\title{
Superdirectional light emission and emission reversal from microcavity arrays
}

\author{
Jakob Kreismann, ${ }^{1, *}$ Jaewon Kim,,${ }^{1}$ Martí Bosch, ${ }^{1}$ Matthias Hein, ${ }^{2}$ Stefan Sinzinger, ${ }^{3}$ and Martina Hentschel ${ }^{1}$ \\ ${ }^{1}$ Institute for Physics, Theoretical Physics II/Computational Physics Group, Technische Universität Ilmenau, \\ Weimarer Straße 25, 98693 Ilmenau, Germany \\ ${ }^{2}$ Department of EI, Electrical Engineering, and Information Technology, RF and Microwave Research Group,Technische Universität Ilmenau, \\ Helmholtzplatz 2, 98693 Ilmenau, Germany \\ ${ }^{3}$ Department of Mechanical Engineering, Optical Engineering Group, Technische Universität Ilmenau, \\ Helmholtzring 1, 98693 Ilmenau, Germany
}

(Received 18 March 2019; published 12 December 2019)

\begin{abstract}
Optical microdisk cavities with certain asymmetric shapes are known to possess unidirectional far-field emission properties. Here, we investigate arrays of these dielectric microresonators with respect to their emission properties resulting from the coherent behavior of the coupled constituents. This approach is inspired by electronic mesoscopic physics where the additional interference effects are known to enhance the properties of the individual system. As an example, we study the linear arrangement of nominally identical Limaçon-shaped cavities and find mostly an increase of the portion of directional emitted light while its angular spread is largely diminished from $20 \mathrm{deg}$ for the single cavity to about $3 \mathrm{deg}$ for a linear array of 10 Limaçon resonators, in fair agreement with a simple array model. Moreover, by varying the intercavity distance, we observe windows of reversion of the emission directionality and superdirectionality that can be interesting for applications like optical sensing or interconnects. We introduce a generalized array factor model that takes the coupling into account.
\end{abstract}

DOI: 10.1103/PhysRevResearch.1.033171

\section{INTRODUCTION}

Optical microdisk cavities have become a target of intense study because of their versatile properties to control light and their unique application potential [1] as high- $Q$ resonators [2], microlasers $[3,4]$, and sensors [5,6]. Moreover, they play an important role as widely accessible experimental realizations of theoretical model systems [7] used in quantum chaos [8-11] and mesoscopic physics [12].

In view of the mutual inspiration of nonlinear dynamics, quantum chaos, antenna theory, and the field of optical microcavities (a paradigm example being the intimate relation between the unstable manifold of the cavity and its far-field emission characteristics $[13,14]$ ), it is suggestive to take further inspiration from mesoscopic physics and its tool box. Besides the wide field of the manipulation of geometric phases $[15,16]$ in dielectric systems [17-19], extension of the single system into array or ensemble structures is known to enhance specific properties in electronic mesoscopic systems [20]. For example, side structures of Aharonov-Bohm peaks in magnetoconductance oscillations were resolved in arrays of electronic microrings due to self-averaging in the composite system [21].

\footnotetext{
*jakob.kreismann@tu-ilmenau.de
}

Published by the American Physical Society under the terms of the Creative Commons Attribution 4.0 International license. Further distribution of this work must maintain attribution to the author(s) and the published article's title, journal citation, and DOI.
In the present work, we want to adopt the concept of arrays to optical microcavities. Our naive expectation and motivation is the possibility of an enhancement of directional emission properties inherent to single deformed optical microdisk cavities, e.g., of the Limaçon shape [14]. To this end, we investigate a linear array (chain) of Limaçon resonators. The shape of each Limaçon resonator is given in two-dimensional polar coordinates $(r, \phi)$ by $r(\phi)=R[1+\delta \cos (\phi)]$, where $R$ and $\delta$ represent the mean radius and the deformation parameter, respectively. The resonator itself is assumed to be purely dielectric with a high refractive index of $n=3.0$ embedded in vacuum, $n_{0}=1$. The deformation parameter is set to $\delta=0.43$, a value known [14] to provide highly directional far fields.

We use MEEP (MIT Electromagnetic Equation Propagation [22]), a free finite-difference time-domain (FDTD) software package for electromagnetic wave simulations, to calculate the normalized resonance frequencies $\Omega=k R=$ $\operatorname{Re}(\omega) R / c$ of the Limaçon resonator, with $\omega$ being the complex frequency and $c$ being the speed of light, the quality factor $Q=-0.5 \operatorname{Re}(\omega) / \operatorname{Im}(\omega)$, the distributions of the electric field component $E_{z}(x, y)$ (modes), and the far-field intensity $I(\theta)$ with far-field angle $\theta$. In the present work, we study wavelength-scale cavities. For this reason, $k R \sim 7$, with $k=$ $2 \pi / \lambda$ being the wave number and $\lambda$ being the wavelength in vacuum. We use $E_{z}$-point-dipole sources (electric field $E$ perpendicular to the cavity) placed off-center to excite the same mode in each cavity and focus on transverse magnetic (TM) polarized modes. In addition, we use COMSOL [23] in order to calculate the eigenfrequency splitting of the resonator array, which results from the exchange of energy among adjacent resonators. 


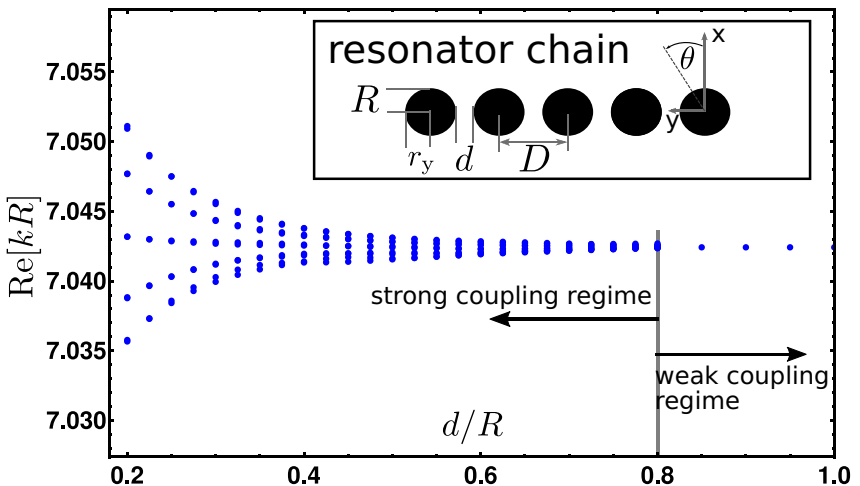

FIG. 1. Frequency splitting of a linear five-element Limaçon resonator array as function of the normalized inter-resonator distance $d / R$ calculated by COMSOL [23]. $D(d)=2 r_{\mathrm{y}}+d$. Two different coupling regimes can be clearly distinguished. The strong-coupling $(d / R<0.8)$ regime exhibits five pairs of eigenfrequencies corresponding to five symmetric and five antisymmetric array modes. The pairs are hardly visible and appear as almost single dots.

We indeed observe the expected enhancement of the farfield emission of Limaçon arrays in comparison to the single resonator due to the coherent operation of all microcavities in the array. We describe this effect in the first part of the paper for the weak (evanescent) coupling regime. However, under certain conditions, we observe the opposite behavior, i.e., a reversal of the main emission direction, or superdirectional emission, as a result of the collective action. We investigate this strong-coupling regime in the second part of the paper and conclude with a summary.

We point out that the excitation scheme used in the MEEP simulations is such that all resonators are excited equally (symmetric configuration, "even mode") close to the eigenfrequency of the single cavity. Since the excitation pulse in each resonator has a finite spectral width that is slightly broader than the spectral splitting of the array modes for the not too small (and experimentally relevant) $d / R$ values considered here, we observe an excitation close to the symmetric array mode with intensity distributions close to that of the single cavity, cf. Supplemental Material [24], and dedicate the following discussion to this case.

\section{ARRAY EMISSION IN THE WEAK-COUPLING REGIME}

We first investigate a row of Limaçon resonators with large inter-resonator distance $d / R \sim 1$. In this regime, the eigenfrequencies do not split (see Fig. 1), indicating that the resonators do not exchange energy. The entire array of resonators can thus be treated as a superposition of individual resonators:

$$
\Phi_{\text {array }}=\sum_{n=1}^{N} \alpha_{n} \psi_{n}\left(x, y-y_{n}\right),
$$

where $\Phi_{\text {array }}$ describes the electric (or magnetic) field distribution of the resonator array, and $\psi_{n}\left(x, y-y_{n}\right)$ are the electric (or magnetic) eigenmodes of a single Limaçon resonator shifted along the $y$ axis by $y_{n}=(n-1) D$. The complexvalued coefficients $\alpha_{n}$ represent the amplitude and phase of the
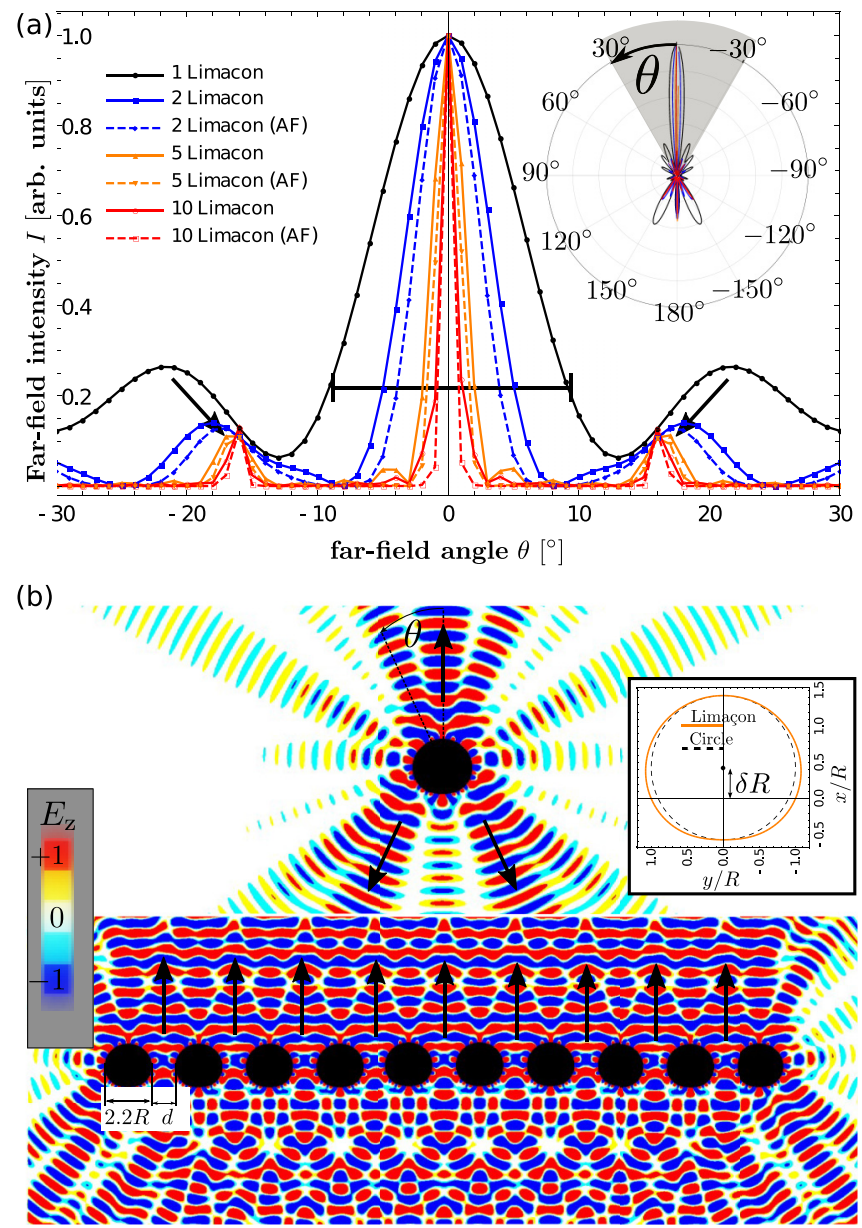

FIG. 2. (a) Intensity plot of the far-field emission from Limaçon cavity arrays. The intensity plot displays the far-field main lobe within the range highlighted by the gray triangular region in the inset (polar plot of the far-field intensity). (b) Field distribution of the $E_{\mathrm{z}}$ component of the electric field of one and 10 Limaçon resonators, respectively. The inter-resonator distance is $d / R=1$. The inset shows the Limaçon shape and for comparison a circle with radius $R$ centered at $x=\delta R$.

$n$th resonator. In order to compute the far fields (fields in the Fraunhofer region) of the array, we use the near-field-to-farfield transformation, a common method in FDTD [25]. From Eq. (1) follows the $z$ component $E_{\mathrm{z}, \text { array }}^{\mathrm{FF}}$ of the electric far field (see Supplemental Material [24]):

$$
E_{z, \text { array }}^{\mathrm{FF}}(\theta) \propto E_{\mathrm{z}, \text { single }}^{\mathrm{FF}}(\theta) \sum_{n=1}^{N} \alpha_{n} e^{-i k D(n-1) \sin \theta},
$$

where $E_{z, \text { single }}^{\mathrm{FF}}$ is the $z$ component of the electric far field of a single resonator and $\theta$ represents the far-field angle; see Fig. 1. The sum on the right-hand side of the equation is also known as the array factor $A F(\theta)$ from antenna theory [26]. The array factor describes the complex-valued far-field pattern of a uniform linear array of point sources. Thus, the total far field results from that of a single resonator multiplied by the array factor. Indeed, our configuration is very similar to antenna arrays. First, we wish to study the difference between the far fields of the single resonator and of an array. Figure 2(a) shows 
the far-field intensity $I \propto\left|E_{\mathrm{z}, \mathrm{array}}^{\mathrm{FF}}(\theta)\right|^{2}$ depending on the farfield angle $\theta$ within the range of \pm 30 degrees for one, two, five, and 10 resonators aligned in a row with constant interresonator distance $d / R=1$. The solid lines are the results from the full FDTD calculation, whereas the dashed lines represent results assuming the array factor model according to Eq. (2). The inset presents the full polar plot of the far-field intensity. The gray region marks the \pm 30 deg range.

The agreement between these results suggests that the resonator array behaves as expected from an antenna array in the weak-coupling regime considered here [27]. As a result, the width of the main lobe decreases with increasing number of array elements (resonators) yielding increased directivity. This feature conforms quantitatively to the array factor model. Specifically, we observe that the width of the main lobe of the far-field emission from 10 Limaçon resonators is reduced by roughly one order of magnitude compared to the singleresonator case. Furthermore, we observe that the position and height of the side lobes varies with the number of elements, saturation at $N>5$ (cf. the black arrows). This behavior results from the array factor as well (see Supplemental Material [24] for more details).

Figure 2(b) displays the normalized $E_{\mathrm{z}}$-field distribution for one and 10 Limaçon resonators, respectively. The far field of one Limaçon resonator (upper panel) is well known and very similar to the results, e.g., in Ref. [28]. The lower panel shows the situation for 10 resonators and reveals a strong emission in the forward direction as indicated by the black arrows. This enhancement arises from the superposition of individual, unperturbed resonator modes of the single Limaçon cavity within the array.

\section{ARRAY EMISSION IN THE STRONG-COUPLING REGIME}

We now investigate the emission of the resonator array for intercavity distances $d / R$ smaller than 1 , i.e., encountering (strong) element coupling. Here, the resonators are close enough to exchange energy by mutual coupling. As a result, the frequencies of the single resonators split and array modes emerge; see Fig. 1 and Supplemental Material [24]. Naturally, the array factor model, Eqs. (1) and (2), remains no longer valid. Due to the coupling, the properties of coupled cavities differ from the isolated case. However, a generalization of this model (generalized array factor model) takes the coupling of the resonators into account by replacing $\psi_{n}$ and $E_{\mathrm{z} \text {,array }}^{\mathrm{FF}}$ of the bare single cavity with the respective quantities $\psi_{n}^{\mathrm{c}}$ and $E_{\mathrm{z} \text {,array }}^{\mathrm{FF}, \mathrm{c}}$ of a coupled cavity region. The far field of the resonator array in the strong coupling regime is modeled by multiplying the far fields of the coupled cavity region with the array factor:

$$
E_{\mathrm{z}, \text { array }}^{\mathrm{FF}, \mathrm{c}}(\theta) \propto E_{\mathrm{z}}^{\mathrm{FF}, \mathrm{c}}(\theta) \sum_{n=1}^{N} \alpha_{n} e^{-i k D(n-1) \sin \theta} .
$$

The generalized array factor model can qualitatively predict the observed array far fields with all features described below while emphasizing the role of coupling.

In order to quantify the directional emission of a resonator array, we introduce the far-field emission ratio $f_{\mathrm{r}}$ that is
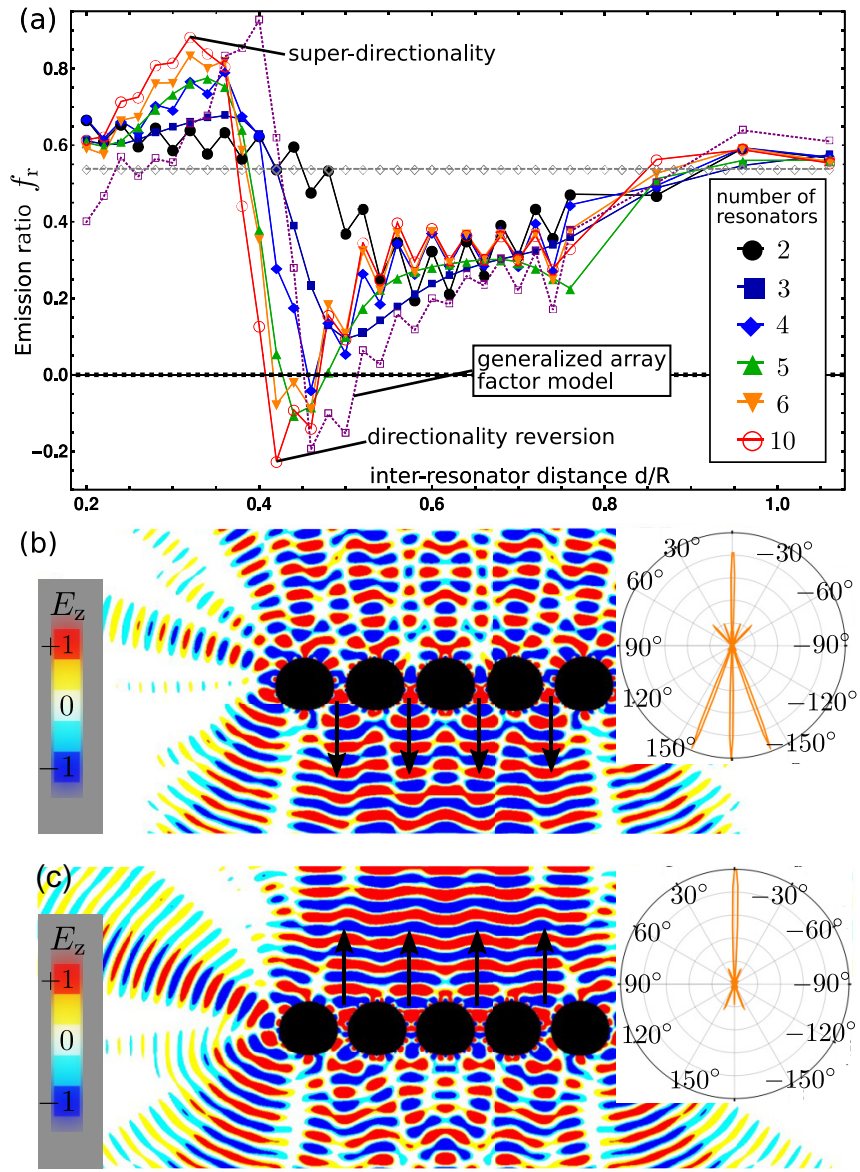

FIG. 3. (a) Emission ratio $f_{\mathrm{r}}$ as function of the inter-resonator distance ratio $d / R$ for arrays with two, three, four, five, six, and 10 resonators. [(b), (c)] Spatial distribution of the normalized electric field amplitude $E_{\mathrm{z}}$ for the 5-resonator array outside the cavities and for distance values (b) $d / R=0.44$ (minimum of $f_{\mathrm{r}}$, reversal of directionality) and (c) $d / R=0.34$ (maximum of $f_{\mathrm{r}}$ - superdirectional light emission in forward direction). Insets show the polar far-field emission. See text for details.

defined by

$$
f_{\mathrm{r}}:=\frac{f_{+}-f_{-}}{f_{+}+f_{-}}
$$

where $f_{+}=\int_{-10^{\circ}}^{10^{\circ}} I(\theta) d \theta$ is the forward directional emission and $f_{-}=\int_{170^{\circ}}^{190^{\circ}} I(\theta) d \theta$ represents the backward directional emission. Emission ratios $f_{\mathrm{r}}$ greater (smaller) than 0 represent forward (backward) directionality, whereas values around 0 indicate balanced forward and backward emissions. By means of the ratio $f_{\mathrm{r}}$, we study the far-field behavior of the resonator array as a function of the inter-resonator distance $d / R$ which is displayed in Fig. 3(a).

\section{REVERSAL OF EMISSION DIRECTIONALITY}

According to Fig. 3(a), we observe that decreasing $d / R$ and hence increasing the coupling strength reduce the emission ratio $f_{\mathrm{r}}$; i.e., less light is emitted into the forward direction. For arrays consisting of more than three resonators, we find the emission ratio $f_{\mathrm{r}}$ to change sign, indicating backward 
emission and thus a reversal of the emission directionality. For the mode chosen and $N=10$ resonators, we find $f_{\mathrm{r}}$ to reach a minimum of around $f_{\mathrm{r}, \min } \approx-0.22$ at a cavity spacing of $d / R \approx 0.42$.

The purple dashed line shows the result of a 10-resonator array obtained with the generalized array factor model ( $\psi_{n}^{\mathrm{c}}$ has been taken from a four-resonator array), which nicely agrees with the results from the full FDTD calculation.

In order to investigate this behavior in more detail, we look at the spatial distribution of the $E_{\mathrm{z}}$ component of the electric field amplitude as depicted in Fig. 3(b). We clearly see a wave propagating downward below the resonator array (resembling plane-wave propagation) as indicated by the black arrows. Above the resonator row, we observe wave propagation in form of an interference pattern that indicates partially destructive interference. The inset displays the entire far-field emission as a polar plot and confirms that more energy is emitted backward than forward.

Assuming coupling between the resonators to be the reason for the directionality reversion, then an interesting question is as follows: Why do five resonators show reversion, but two resonators do not? Following the $f_{\mathrm{r}}$ curve of two resonators in Fig. 3(a), we observe that the emission ratio decreases a bit with decreasing $d / R$ values but its minimum remains positive. This slight decrease of $f_{\mathrm{r}}$ indicates an increase of the energy emitted into the backward direction. It turns out that directionality reversion is already present for two resonators but it is masked by the emission from the edges of the array (see Supplemental Material [24]). The directionality reversion arises from the coupling regions (in-between space of two resonators) and it needs a certain number of these coupling regions to overcome the effect of the emission from the edge regions.

\section{SUPERDIRECTIONAL LIGHT EMISSION FROM LINEAR ARRAYS}

By decreasing the distance $d / R$ between the resonators further, the emission ratio changes sign and reaches a maximum $f_{\mathrm{r}, \max } \approx 0.89$ at $d / R=0.32$ for $N=10$ resonators. This maximum is even higher than the value found at the element distance $d / R \approx 1.0$. It indicates that the coherent modal action of the resonator array results in a superdirectional forward emission of light. This is illustrated in Fig. 3(c). Contrary to Fig. 3(b), we now find (almost) planar wave propagation into the forward direction. The interference pattern visible below the resonator row indicates destructive interference and hence reduced energy flow into the backward direction, as confirmed by the inset showing the full polar plot.

We point out that the emission patterns in Figs. 2(b) and 3 (c) look similar as both correspond to values $f_{\mathrm{r}}>0$. Notice, however, that the planar-wave-type propagation (destructive interference) in forward (backward) direction are even more pronounced for the strong coupling case shown in Fig. 3(c).

The gray dashed line in Fig. 3(a) corresponds to the (standard) array factor model [Eq. (2)] with $N=10$ resonators. It represents the case of weakly coupled resonators and shows a constant $f_{\mathrm{r}}$ value because of $|A F(\theta)|^{2}=|A F(\pi+\theta)|^{2}$ and $E_{\mathrm{z}, \text { single }}^{\mathrm{FF}}(\theta)$ being independent from $d / R$.

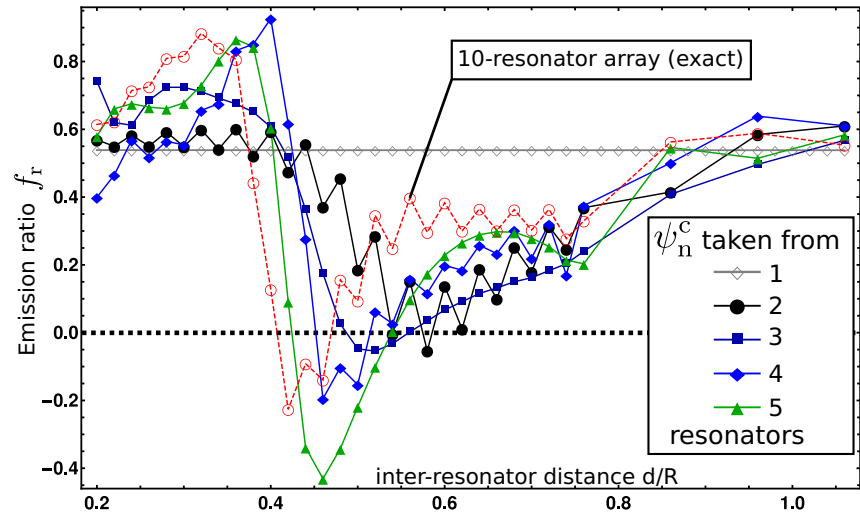

FIG. 4. Results of the emission ratio $f_{\mathrm{r}}$ calculated from the generalized array factor model for $N=10$, cf. Eq. (3), where $\psi_{n}^{\mathrm{c}}$ was taken from one-, two-, three-, four-, and five-element resonator arrays, respectively. The dashed red line represents the "exact" emission ratio of a 10-element resonator array.

\section{GENERALIZED ARRAY FACTOR MODEL}

We now study the generalized array factor model in more detail. In this context, an interesting question is as follows: Is it possible to predict the features of a 10-resonator array with the aid of the generalized array factor model utilizing the coupling features of a two- or three-resonator array?

To this end, we compute the emission ratio for a 10 resonator array based on the far fields obtained from the generalized array factor model where the $\psi_{n}^{\mathrm{c}}$ have been taken from one-, two-, three-, four-, and five-element resonator arrays, respectively (see Supplemental Material [24]). The corresponding results are shown in Fig. 4 with a comparison to the exact emission ratio of a 10-resonator array. The case of $\psi_{n}^{\mathrm{c}}$ taken from one resonator is identical to the array factor model of uncoupled resonators, as described by Eq. (2).

The emission ratio with $\psi_{n}^{\mathrm{c}}$ taken from two resonators displays very weak directionality reversion and very weak superdirectionality. At least, one could guess that such features exist. As pointed out earlier, the directionality reversion feature is already present for two resonators but it is masked by the emission from the edge regions. The best agreement with the exact curve is obtained for the case with $\psi_{n}^{\mathrm{c}}$ taken from four resonators. Interestingly, the results with $\psi_{n}^{\mathrm{c}}$ taken from five resonators show too much directionality reversion compared to the exact result. The reason for this is that we neglected the role of the amplitudes $\alpha_{n}$. For reasons of simplicity, the $\alpha_{n}$ have been set to unity, which is not correct if the array gets larger, because of the amplitude distribution within the array mode with high amplitudes for central resonators and low amplitudes for edge resonators.

The generalized array factor model can qualitatively predict the emission ratio of a 10-resonator array and reveals that the reversion of the directionality arises from the coupling of the single resonator modes. It cannot be modeled by a proper superposition of the single resonator modes [as described in Eq. (2)]. Thus, the coupling between the resonators plays an essential role. This implies immediately that the reversed and superdirectional emission will be resonance dependent; see the Supplemental Material [24]. A complementary 
interpretation of our findings based on (generalized) phasespace arguments [14] is in progress and will be the subject of a subsequent study.

\section{CONCLUSION}

Our FDTD calculations revealed that the directionality of the emission of photonic devices can be considerably enhanced using array structures, both concerning the fraction of the directionally emitted light and its angular spread. Moreover, the main emission direction of the cavity ensemble can be inverted such that light is mostly emitted into the direction opposite the one of the singly cavity. We argue that this effect arises from the coupling of the individual resonators. The directionality properties depend strongly on (i) the normalized distance $d / R$ between the resonators and (ii) the properties of the individual resonant modes. Furthermore, the directionality reversion behavior depends on the number of the resonators in the array and emerges only if their number is larger than three.

\section{ACKNOWLEDGMENTS}

This work was partly supported by Emmy-Noether programme of the German Research Foundation (DFG). Furthermore, we thank Christoph Wagner from the Department of Advanced Electromagnetics for fruitful discussions.
[1] K. J. Vahala, Nature (London) 424, 839 (2003).

[2] C. P. Michael, K. Srinivasan, T. J. Johnson, O. Painter, K. H. Lee, K. Hennessy, H. Kim, and E. Hu, Appl. Phys. Lett. 90, 051108 (2007).

[3] S. L. McCall, A. F. J. Levi, R. E. Slusher, S. J. Pearton, and R. A. Logan, Appl. Phys. Lett. 60, 289 (1992).

[4] T. Harayama and S. Shinohara, Laser Photon. Rev. 5, 247 (2011).

[5] A. M. Armani, R. P. Kulkarni, S. E. Fraser, R. C. Flagan, and K. J. Vahala, Science 317, 783 (2007).

[6] X. Xu, W. Chen, G. Zhao, Y. Li, C. Lu, and L. Yang, Light: Sci. Appl. 7, 62 (2018).

[7] H. Cao and J. Wiersig, Rev. Mod. Phys. 87, 61 (2015).

[8] H.-J. Stckmann, Quantum Chaos (Cambridge University Press, Cambridge, UK, 1999).

[9] J. U. Nöckel and A. D. Stone, Nature (London) 385, 45 (1997).

[10] J. Yang, S.-B. Lee, S. Moon, S.-Y. Lee, S. W. Kim, T. T. A. Dao, J.-H. Lee, and K. An, Phys. Rev. Lett. 104, 243601 (2010).

[11] J. Wiersig, A. Eberspächer, J.-B. Shim, J.-W. Ryu, S. Shinohara, M. Hentschel, and H. Schomerus, Phys. Rev. A 84, 023845 (2011).

[12] S. Datta, Physics Today (Cambridge University Press, Cambridge, UK, 1995).

[13] S.-Y. Lee, J.-W. Ryu, T.-Y. Kwon, S. Rim, and C.-M. Kim, Phys. Rev. A 72, 061801(R) (2005).

[14] J. Wiersig and M. Hentschel, Phys. Rev. Lett. 100, 033901 (2008).

[15] M. V. Berry, Proc. R. Soc. London A 392, 45 (1984).

[16] M. Nakahara, Geometry, Topology, and Physics (IOP, Bristol, UK, 1990).
[17] K. Y. Bliokh, A. Niv, V. Kleiner, and E. Hasman, Nat. Photon. 2, 748 (2008).

[18] L. B. Ma, S. L. Li, V. M. Fomin, M. Hentschel, J. B. Götte, Y. Yin, M. R. Jorgensen, and O. G. Schmidt, Nat. Commun. 7, 10983 (2016).

[19] J. Kreismann and M. Hentschel, Europhys. Lett. 121, 24001 (2018).

[20] E. Dupont, P. B. Corkum, H. C. Liu, M. Buchanan, and Z. R. Wasilewski, Phys. Rev. Lett. 74, 3596 (1995).

[21] J. Nitta, H. Takayanagi, and S. Calvet, Microelectron. Eng. 47, 85 (1999).

[22] A. F. Oskooi, D. Roundy, M. Ibanescu, P. Bermel, J. D. Joannopoulos, and S. G. Johnson, Comput. Phys. Commun. 181, 687 (2010).

[23] Wave Optics Module User's Guide, COMSOL Multiphysics® v. 5.4. COMSOL AB, 2018, http://www.comsol.com.

[24] See Supplemental Material at http://link.aps.org/supplemental/ 10.1103/PhysRevResearch.1.033171 for details about the array modes, the array factor model, and the generalized array factor model.

[25] U. S. Inan and R. A. Marshall, Numerical Electromagnetics (Cambridge University Press, Cambridge, UK, 2011).

[26] C. A. Balanis, Antenna Theory: Analysis and Design, 4th ed. (Wiley, New York, 2016), p. 1104.

[27] The coefficients $\alpha_{n}$ have been all set to unity because there is no mutual coupling between the resonators and all resonators have been excited equally.

[28] Q. H. Song, L. Ge, J. Wiersig, J.-B. Shim, J. Unterhinninghofen, A. Eberspächer, W. Fang, G. S. Solomon, and H. Cao, Phys. Rev. A 84, 063843 (2011). 